\title{
28 Research Square \\ Individualized Growth Hormone Treatment - Using Two Different Prediction Models in a Clinical Setting
}

Helena-Jamin Ly ( $\square$ ly.helena@gmail.com )

University of Gothenburg

Anders Lindberg

University of Gothenburg

Hans Fors

University of Gothenburg

Jovanna Dahlgren

University of Gothenburg

\section{Research Article}

Keywords: Growth hormone treatment, prediction model, growth outcome

Posted Date: January 3rd, 2022

DOI: https://doi.org/10.21203/rs.3.rs-1178621/v1

License: (9) This work is licensed under a Creative Commons Attribution 4.0 International License.

Read Full License 


\section{Abstract}

\section{Background}

Diagnosing growth hormone deficiency (GHD) can be challenging; hence, prediction models on growth outcome from growth hormone $(\mathrm{GH})$ treatment have shown to be useful. We aim to compare the accuracy of the more readily available KIGS (Pfizer International Growth Study) prediction model to the previously clinically validated Gothenburg model.

\section{Methods}

Prepubertal children with GHD who started GH treatment at Queen Silvia Children's Hospital between 2004 and 2016 were considered for the study. Exclusion criteria were short stature due to syndrome, chronic disease, oncology disease, or known bad adherence. Growth predictions were made according to the Gothenburg model and the KIGS model. Growth data from birth until one year after start of GH treatment were collected from medical charts. Predicted height and observed height were then compared.

\section{Results}

A total of 123 children, 47 girls (38\%) and 76 boys (62\%) were included, with a mean age of $5.71( \pm 1.81$ SD) years at start of $\mathrm{GH}$ treatment. The Pearson correlation of predicted first-year growth versus growth outcome were $r=0.990$ for the Gothenburg model and $r=0.991$ for the KIGS model. Studentized residuals were $0.10 \pm 0.81 \mathrm{SD}$ and $0.03 \pm 0.96 \mathrm{SD}$, respectively, for the models. The comparison between the two models showed $r=0.995$.

\section{Conclusion}

The Gothenburg model and the KIGS model are equally accurate at predicting height outcome from GH treatment for our study cohort. We therefore promote the use of either model in clinical settings.

\section{Background}

Diagnosing growth hormone deficiency (GHD) is a multifaceted process that requires clinical, auxological, biochemical, and radiological data [1]. A gold standard diagnostic test for GHD is lacking. This makes it difficult in some cases to make a correct diagnosis, and to identify children who will benefit from recombinant growth hormone (GH).

Historically, evaluating the effect of $\mathrm{GH}$ treatment has been done retrospectively by measuring the height gain or metabolic response to $\mathrm{GH}$ treatment. Consequently, this approach might lead to short children being treated unnecessarily as they don't respond well enough to treatment, and with possible long-term risks. Bang et al. found that in a cohort of $173 \mathrm{GHD}$ patients $28 \%$ were poor responders, defined here as a first-year delta growth of less than 0.5 standard deviation scores (SDS) [2]. To avoid treating poor responders, an approach where the clinician can predict outcome before start of treatment would be 
useful. Therefore, prediction models have been mathematically constructed where variables called predictors have been included using regression analyses. By using the individualized predictors, the outcome gives a prognostic value for the first-year growth for the individual patient. Depending on the expected growth, a decision can be made as to whether GH treatment should be considered. The first-year

growth response to $\mathrm{GH}$ treatment has in fact been shown to be a good indicator of the growth response in the following years of treatment [3-5].

Several prediction models have been constructed worldwide based on different predictors $[3,4,6]$. All have used large but retrospective treatment data from patients to construct the model, and then a smaller group of patients to validate the model [7]. The usefulness of a model is determined by how well it works in practice and not the level of statistical significance; therefore, validation of a model is very crucial, using data from a group of people who fulfill the inclusion criteria for the model but whose data were not used to derive it initially [8]. In our study we focus on two different published prediction models.

\section{The KIGS prediction model}

Post-marketing surveillance studies of recombinant human GH therapy, such as the KIGS (Pfizer International Growth study), have accumulated extensive data concerning the characteristics and growth outcomes of approximately 83,000 children with various causes of short stature.

Data from a cohort of 593 prepubertal children from the database with idiopathic GHD were obtained to create two KIGS prediction models for prepubertal children [3]. The variables included in the predictions were as follows: birth weight, gestational age (GA), gender, parental heights, chronological age, height and weight at start of treatment, and weekly GH dose. In addition to those variables mentioned, one model also included stimulated GH peak. An internal validation of the first-year response showed a mean studentized residual of $0.1 \pm 1.3$ SDS for the model that included stimulated maximum GH peak data and $0.0 \pm 1.2$ SDS for the model without stimulated maximum GH peak.

\section{The Gothenburg.prediction models}

The first Gothenburg prediction models were published by Albertsson-Wikland et al. in 2000 [4]. The specific model called " $\mathrm{GH}_{24 \mathrm{~h}}$ combined with early growth" showed the best accuracy, with a residual SD of 0.19 SDS at internal validation. This model consists of variables such as maximum $\mathrm{GH}$ peak from spontaneous profile; parental heights; birth weight and length; gender; GA; and auxology at one year, two years, and one year pretreatment, and at start of $\mathrm{GH}$ treatment.

The approach with prediction model-guided treatment initiation has been in use for over a decade in the pediatric endocrine department at Queen Silvia Children's Hospital, Gothenburg, Sweden. A recent validation of the model in use [4] has been clinically validated, showing that only $2 \%$ of the 121 prepubertal children who started treatment based on the prediction model between 2004 and 2016 grew less than 0.5 SDS in a year and were defined as poor responders [9]. 
The previously published data assure that prediction models are a helpful clinical tool to avoid unnecessary GH treatment [9], but to date few clinicians seem to use them in the decision-making on whether to start treatment or not. To enhance the use of prediction models, we aim to compare the accuracy of the KIGS prepubertal prediction model, which is more readily available, to the previously validated Gothenburg prediction model.

\section{Methods}

All consecutive prepubertal children who started GH treatment between 1 January 2004 and 31 December 2016 at Queen Silvia Children's Hospital in Gothenburg were considered for the study. Pubertal children (defined as Tanner breast stage $>1$ in girls and testis $>3 \mathrm{ml}$ in boys), and children with oncological disease, chronic illness, or known bad adherence were excluded. So were children born preterm under gestational week 30 and children born small for gestational age (SGA, defined here as birth weight or length $<-2.5$ SDS) [10].

For all 123 prepubertal children, 47 girls (38\%) and 76 boys (62\%) who were included, the following data were retrieved from medical charts: parental heights; gender; GA; birth weight and birth length; spontaneous maximum GH peak; and weight and length at one year, two years, one year prior to treatment, and at treatment start. Outcome growth data after one year of treatment were also assembled.

From these data, new growth predictions were made according to the prediction models from Gothenburg and KIGS $[3,4]$. The Gothenburg prediction model consists of several models, and specifically, the model called $\mathrm{GH}_{24 \mathrm{~h}}$ combined with early growth was used [4].

The KIGS model that includes stimulated GH peak has an upper limitation of $10 \mu \mathrm{g} / \mathrm{L}$, which means that the patients $(n=14)$ with higher GH peak were instead predicted using the model without the GH peak variable. Two girls could not be predicted according to any of the KIGS models because of their age exceeding 10 years, which is an upper age limitation for the models.

Six patients with a birth weight between -2 SDS and -2.5 SDS were nonetheless included in the study, although unlike the Gothenburg model, the KIGS original cohort did not include SGA <-2SD.

\section{Growth}

Growth data from birth to school age were retrieved from child welfare centers. Birth weight SDS was adjusted for GA on the basis of work by Niklasson et al [10] while other weight SDS are based on Freeman et al [11].

Height was measured at the pediatric endocrine outpatient clinic using an Ulmer stadiometer, and body weight was measured to the nearest $0.1 \mathrm{~kg}$ using an electronic step scale (SECA 701, Germany). All anthropometric data after one year of treatment were obtained within $12 \pm 2$ months. 
The KIGS model predicts a first-year growth in $\mathrm{cm} /$ year, while the Gothenburg prediction model predicts in SDS according to the childhood component of the infancy, childhood, and puberty (ICP) growth model of Karlberg et al. [12]. The latter predictions were thus converted to $\mathrm{cm} / y e a r$ to be able to make a comparison with the KIGS results. Parental heights are presented according to Karlberg et al. [13].

One of the parental heights was $205 \mathrm{~cm}$, which exceeded the upper limitation of $200 \mathrm{~cm}$ applicable for the KIGS prediction model. This one parental height was reduced to $200 \mathrm{~cm}$ to be able to fit in the prediction model.

\section{Hormonal analyses}

GH maximum from the 12-hour and 24-hour spontaneous profiles were analyzed using a fluoroimmunometric assay with monoclonal antibodies, and the WHO IRP 80/505 Standard AutoDELFIA hGH used expressed GH in mU/L (PerkinElmer Life and Analytical Sciences, Turku, Finland). This method was changed to a chemiluminescent immunoassay IDS-iSYS (Immunodiagnostic Systems Holdings, Tyne and Wear, UK) from 16 June 2014, expressing GH in $\mu \mathrm{g} / \mathrm{L}$. The transformation formula IDS-iSYS = AutoDELFIA/2.6 was used.

Kriström et al. found that the night GH peak was the most predictive from the GH profile, and therefore, it was later shortened from 24 hours to 12 hours nighttime [14]. Duchen et al. found that stimulated maximum GH peak could reliably be replaced by maximum GH peak from profile [15]. Thus, for the KIGS model that included maximum GH peak, we used spontaneous $\mathrm{GH}$ peak from profile, which is standard at our clinic.

\section{Statistics}

All basic statistics are presented by mean and standard deviations.

Correlations are calculated using Pearson correlation coefficient.

To detect outliers and estimate the accuracy of the prediction models, studentized residual plots where performed.

All statistical calculations were done using SAS 9.4 for Windows.

\section{Results}

\section{Background characteristics}

The patient characteristics are shown in Table 1.

\section{Table 1. Patient characteristics}




\begin{tabular}{|llll|}
\hline Variable & N & Mean & SDS \\
\hline Gestational age (weeks) & 123 & 39.07 & 2.26 \\
\hline Birth weight (SDS) & 123 & -0.29 & 1.08 \\
\hline Maternal height (SDS) & 123 & -0.42 & 1.02 \\
\hline Paternal height (SDS) & 123 & -0.41 & 1.13 \\
\hline Mid-parental height (SDS) & 123 & -0.42 & 0.88 \\
\hline GH maximum peak ( $\mu \mathrm{g} / \mathrm{L})$ & 123 & 6.76 & 3.19 \\
\hline At start of GH & & & \\
\hline Age (years) & 123 & 5.71 & 1.81 \\
\hline Height (cm) & 123 & 103.04 & 10.61 \\
\hline Height (SDS) & 123 & -2.40 & 0.65 \\
\hline Weight (SDS) & 123 & -1.74 & 0.89 \\
\hline GH dose (mg/kg/day) & 123 & 0.03 & 0.01 \\
\hline
\end{tabular}

The mean age at treatment start for the study cohort was 5.71 years \pm 1.81 SDS, which was earlier than the Gothenburg model cohort with mean age 9.2 years \pm 2.5 SDS and KIGS model cohort with mean age $7.3 \pm 2.1$ SDS. The mean spontaneous maximum GH peak for the study cohort was $6.76 \mu \mathrm{g} / \mathrm{L} \pm 3.19$ SDS, compared to the Gothenburg model cohort with mean spontaneous maximum GH peak of $15 \mu \mathrm{g} / \mathrm{L}$ $\pm 9.1 \mathrm{SDS}$ and the KIGS model cohort with mean stimulated maximum GH peak of $5.6 \mu \mathrm{g} / \mathrm{L} \pm 2.8 \mathrm{SDS}$.

\section{Growth outcome}

The mean height for the patients after one year of GH treatment was $113.0 \mathrm{~cm} \pm 10.3$ SDS, while the Gothenburg model predicted $112.9 \mathrm{~cm} \pm 10.5$ SDS, and the KIGS model predicted $112.7 \mathrm{~cm} \pm 9.9$ SDS.

\section{Correlations}

The Pearson correlation coefficient between observed height after one year of treatment compared to the Gothenburg predictions and the KIGS predictions are $r=0.990, p=<0.0001$, and $r=0.991, p=<0.0001$, respectively (see Figs. 1 and 2).

\section{Studentized residuals}

Figure 3 shows the plotted individualized studentized residuals in relation to predicted growth responses using the Gothenburg model, and Figure 4 shows the relation to the KIGS models, with studentized residuals of $0.10 \pm 0.81 S D$ and $0.03 \pm 0.96 S D$, respectively. 
Patient A was under-predicted in both models, with much higher observed height after one year of treatment. She was born to short parents (father $155 \mathrm{~cm}$ and mother $153 \mathrm{~cm}$ ) and had an older sister (not included in the study) with similar growth pattern, who also responded well to $\mathrm{GH}$ treatment, making them suspicious for a hereditary form of $\mathrm{GH}$-sensitive short stature.

\section{Comparing the models}

The comparison between the models shows $r=0.995, p=<0.0001$ (see Fig. 5).

\section{Discussion}

The application of a prediction model for $\mathrm{GH}$ treatment outcome has previously shown to be of benefit in a clinical setting, where only $2 \%$ of patients $(2 / 123)$ were found to be poor responders to $\mathrm{GH}$ treatment when the prediction model was used in the decision-making on whether to start treatment [9]. Prediction models are also useful in growth monitoring, as a patient who deviates from the predicted growth can indicate low/bad adherence or even short stature due to diagnosis other than GHD [16-18].

It is important to find a model that is applicable to your specific cohort for best results, and therefore, we made a clinical validation and comparison of two available models, namely, the Gothenburg and the KIGS prediction models. To our knowledge, a clinical comparison between the two prediction models has not previously been performed.

We have shown with this clinical validation that both models show an equally great accuracy with correlation to observed height being 0.99 for both models without signs of bias. These results are better than expected, as it is known that prediction models often do not perform as well on new cohorts. In the validation of the KIGS model by Ridder et al. where 136 Dutch patients were included, the results showed over-fitting whereby low predictions tended to be underestimated, while high predictions tended to be overestimated[19]. In the validation by Vosahlo et al. [20] for the same model, where 38 patients from Austria were included, no significant difference between the observed and predicted first-year growth was found for the model that included GH peak, while for the model without GH peak the predictions were found to be significantly underestimated.

Our cohort has the same inclusion criteria as the original cohorts for the prediction models, with an exception for the few patients who were born too small for the KIGS model but fulfilled criteria to get a prediction from the Gothenburg model. Some basic parameters differed; for example, the spontaneous maximum GH peaks had a lower mean for the study group and a lower age at start of treatment, compared to both models, but the predictions were still very accurate.

The KIGS model has benefits of being more user-friendly, with higher access through the web-based online iGRO software [21] and fewer variables needing to be inserted. The predictions are also doseadjusted. The original prediction cohort was a heterogeneous group assembled from various 
investigators from many countries. Our validation of this model fulfilled the criteria of geographical external validation (that is, validity of predictions for patients in another geographical area) [22].

The Gothenburg model predicts on a standard dose of $30 \mu \mathrm{g} / \mathrm{kg} /$ day but has wider inclusion criteria without upper limitation of maximum GH peaks or parental heights. The Gothenburg model has previously been validated clinically, and by using a first-year growth outcome prediction cut-off of 0.7 SDS, unnecessary treatment of possible poor responders to $\mathrm{GH}$ treatment could be avoided [9]. The use of cut-off values for model predictions to guide decision-making is important to enhance usage, rather than only providing predicted probability [22]. To validate a cut-off for a first-year prediction for the KIGS model would be of great interest to enhance the usage.

We are pleased to find that both models are equally accurate and encourage the usage of prediction models in clinical settings. We are convinced that it will improve cost-benefit of $\mathrm{GH}$ treatment by avoiding unnecessary treatment for possible poor responders.

\section{Conclusion}

Both the Gothenburg model and the KIGS model are equally accurate at predicting height outcome from $\mathrm{GH}$ treatment for our study cohort. We therefor promote the usage of either model in a clinical setting.

\section{List Of Abbreviations}

$\mathrm{GA}=$ Gestational age

$\mathrm{GH}=$ Growth hormone

GHD $=$ Growth hormone deficiency

SDS $=$ Standard deviation scores

\section{Declarations}

\section{Ethics approval and consent to participate}

The study was performed according to the Helsinki declaration, and the research protocol was approved by the Gothenburg Research Ethics Committee (ref: 171-18). The Gothenburg Research Ethics Committee (ref: 171-18) waived the requirements of consent.

\section{Consent for publication}

Not applicable

\section{Availability of data and materials}


The datasets used and analyzed during the current study are available from the corresponding author on reasonable request.

\section{Competing interests}

The authors declare that they have no competing interests.

\section{Funding}

This study was supported by a grant from ALF Gothenburg (ALFGBG-719711).

\section{Authors' contributions}

$\mathrm{HL}$ as corresponding author contributed to the study design and collection of data, and drafted the manuscript. HF made substantial contributions to the design of the article and data interpretation, and substantively revised it. AL contributed to the design, made the analyses, and interpreted the data. JD contributed to the design, interpreted the data, and was involved in the writing of the manuscript.

All authors read and approved the final manuscript.

\section{Acknowledgments}

Not applicable

\section{References}

1. Israel E, Attie KM, Bengtsson BA, Blethen SL, Blum W, Cameron F, et al. Consensus guidelines for the diagnosis and treatment of growth hormone $(\mathrm{GH})$ deficiency in childhood and adolescence: Summary statement of the GH Research Society. J Clin Endocr Metab. 2000;85(11):3990-3.

2. Bang P, Bjerknes R, Dahlgren J, Dunkel L, Gustafsson J, Juul A, et al. A comparison of different definitions of growth response in short prepubertal children treated with growth hormone. Horm Res Paediatr. 2011;75(5):335-45.

3. Ranke MB, Lindberg A, Chatelain P, Wilton P, Cutfield W, Albertsson-Wikland K, et al. Derivation and validation of a mathematical model for predicting the response to exogenous recombinant human growth hormone $(\mathrm{GH})$ in prepubertal children with idiopathic GH deficiency. KIGS International Board. Kabi Pharmacia International Growth Study. J Clin Endocrinol Metab. 1999;84(4):1174-83.

4. Wikland KA, Kristrom B, Rosberg S, Svensson B, Nierop AF. Validated multivariate models predicting the growth response to $\mathrm{GH}$ treatment in individual short children with a broad range in $\mathrm{GH}$ secretion capacities. Pediatr Res. 2000;48(4):475-84.

5. Dahlgren J, Kristrom B, Niklasson A, Nierop AF, Rosberg S, Albertsson-Wikland K. Models predicting the growth response to growth hormone treatment in short children independent of GH status, birth size and gestational age. BMC Med Inform Decis Mak. 2007;7:40. 
6. Schonau E, Westermann F, Rauch F, Stabrey A, Wassmer G, Keller E, et al. A new and accurate prediction model for growth response to growth hormone treatment in children with growth hormone deficiency. Eur J Endocrinol. 2001;144(1):13-20.

7. Ranke MB, Wit JM. Growth hormone - past, present and future. Nat Rev Endocrinol. 2018;14(5):285300.

8. Altman DG, Royston P. What do we mean by validating a prognostic model? Stat Med. 2000;19(4):453-73.

9. Ly HJ, Fors H, Nilsson S, Dahlgren J. A prediction model could foresee adequate height response in children eligible for growth hormone treatment. Acta Paediatrica. 2021.

10. Niklasson A, Ericson A, Fryer JG, Karlberg J, Lawrence C, Karlberg P. An update of the Swedish reference standards for weight, length and head circumference at birth for given gestational age (1977-1981). Acta Paediatr Scand. 1991;80(8-9):756-62.

11. Freeman JV, Cole TJ, Chinn S, Jones PR, White EM, Preece MA. Cross sectional stature and weight reference curves for the UK, 1990. Archives of disease in childhood. 1995;73(1):17-24.

12. Karlberg J, Engstrom I, Karlberg P, Fryer JG. Analysis of linear growth using a mathematical model. I. From birth to three years. Acta Paediatr Scand. 1987;76(3):478-88.

13. Karlberg P, Taranger J, Engstrom I, Karlberg J, Landstrom T, Lichtenstein H, et al. I. physical growth from birth to 16 years and longitudinal outcome of the study during the same age period. Acta Paediatr Scand Suppl. 1976(258):7-76.

14. Kristrom B, Lofqvist C, Rosberg S, Albertsson Wikland K. Effect of spontaneous GH secretion and the $\mathrm{GH}$ sampling period on the accuracy of models for predicting growth responses to $\mathrm{GH}$ treatment. J Clin Endocrinol Metab. 2001;86(10):4963-4.

15. Duchen K, Lindberg A, Kiplok K, Kristrom B. Using a spontaneous profile rather than stimulation test makes the KIGS idiopathic growth hormone deficiency model more accessible for clinicians. Acta Paediatr. 2017;106(9):1481-6.

16. Wit JM, Ranke MB, Albertsson-Wikland K, Carrascosa A, Rosenfeld RG, Van Buuren S, et al. Personalized approach to growth hormone treatment: clinical use of growth prediction models. Horm Res Paediatr. 2013;79(5):257-70.

17. Ranke MB. New paradigms for growth hormone treatment in the 21st century: prediction models. J Pediatr Endocrinol Metab. 2000;13 Suppl 6:1365-9.

18. Kristrom B, Wikland KA. Growth prediction models, concept and use. Horm Res. 2002;57 Suppl 2:6670.

19. de Ridder MA, Stijnen T, Hokken-Koelega AC. Validation and calibration of the Kabi Pharmacia International Growth Study prediction model for children with idiopathic growth hormone deficiency. J Clin Endocrinol Metab. 2003;88(3):1223-7.

20. Vosahlo J, Zidek T, Lebl J, Riedl S, Frisch H. Validation of a mathematical model predicting the response to growth hormone treatment in prepubertal children with idiopathic growth hormone deficiency. Horm Res. 2004;61(3):143-7. 
21. Loftus J, Lindberg A, Aydin F, Gomez R, Maghnie M, Rooman R, et al. Individualised growth response optimisation (iGRO) tool: an accessible and easy-to-use growth prediction system to enable treatment optimisation for children treated with growth hormone. J Pediatr Endocrinol Metab. 2017;30(10):1019-26.

22. Steyerberg EW, Moons KG, van der Windt DA, Hayden JA, Perel P, Schroter S, et al. Prognosis Research Strategy (PROGRESS) 3: prognostic model research. PLoS Med. 2013;10(2):e1001381.

\section{Figures}

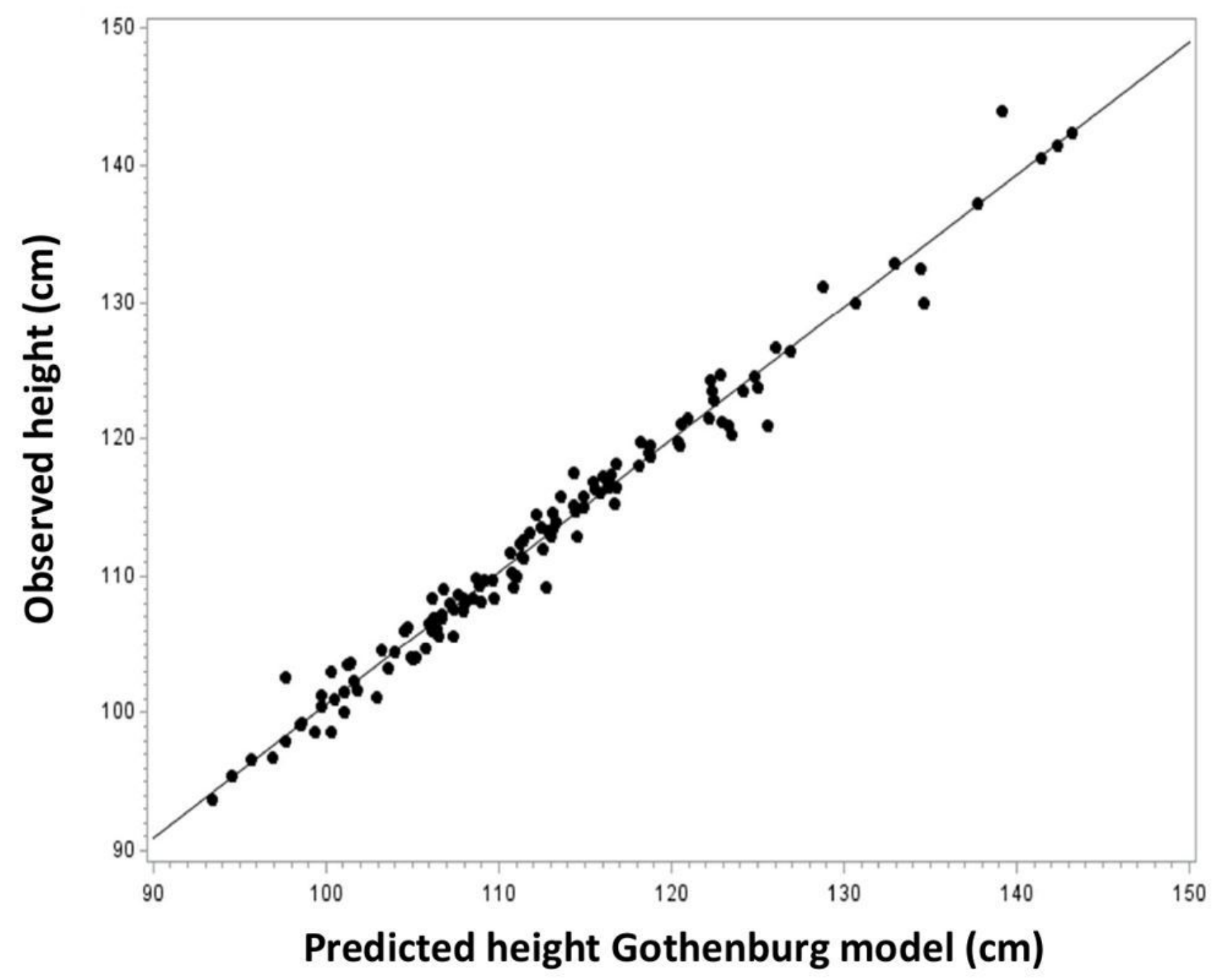

Figure 1

$Y$-axis shows the height outcome after one year of growth hormone treatment versus the predicted height outcome according to the Gothenburg prediction model on the $x$-axis, with a Pearson correlation 
coefficient of $r=0.990, p=<0.0001$.

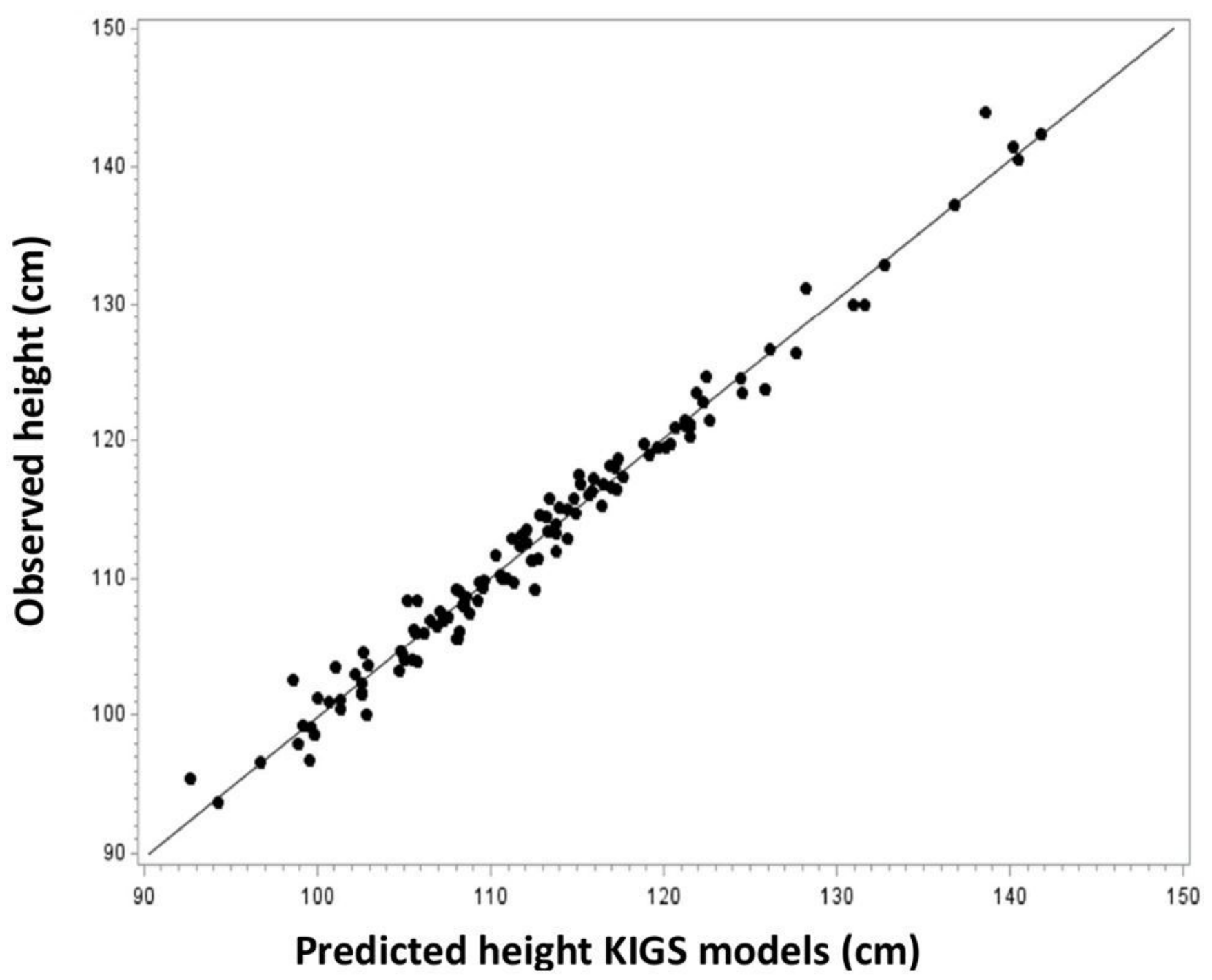

Figure 2

$Y$-axis shows the height outcome after one year of growth hormone treatment versus the predicted height outcome according to the KIGS model on the $x$-axis, with a Pearson correlation coefficient of $r=0.991, p=$ $<0.0001$. 


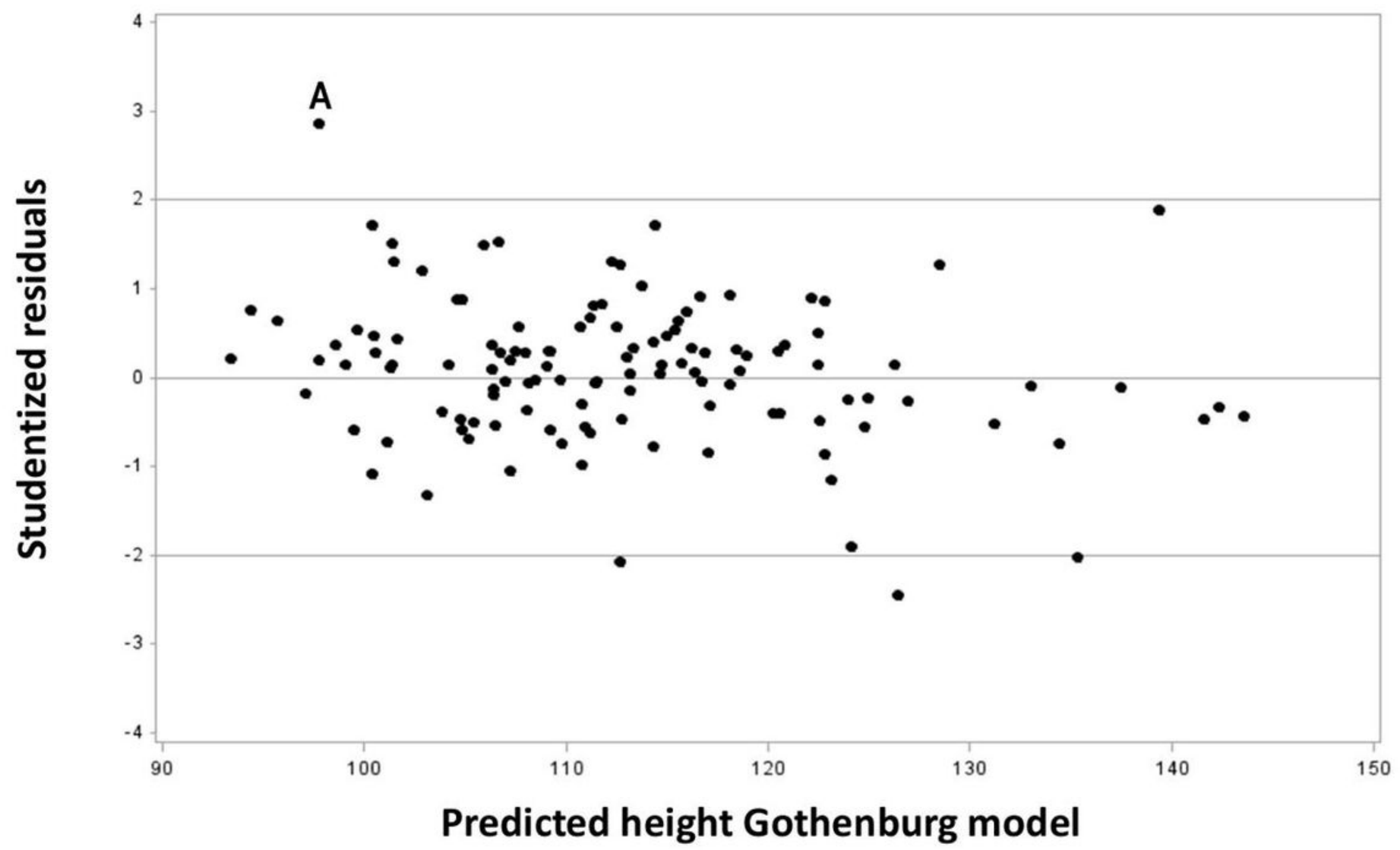

Figure 3

Individual studentized residuals in relation to first-year prediction according to the Gothenburg model. Patient $A$ under-predicted with a high studentized residual. 


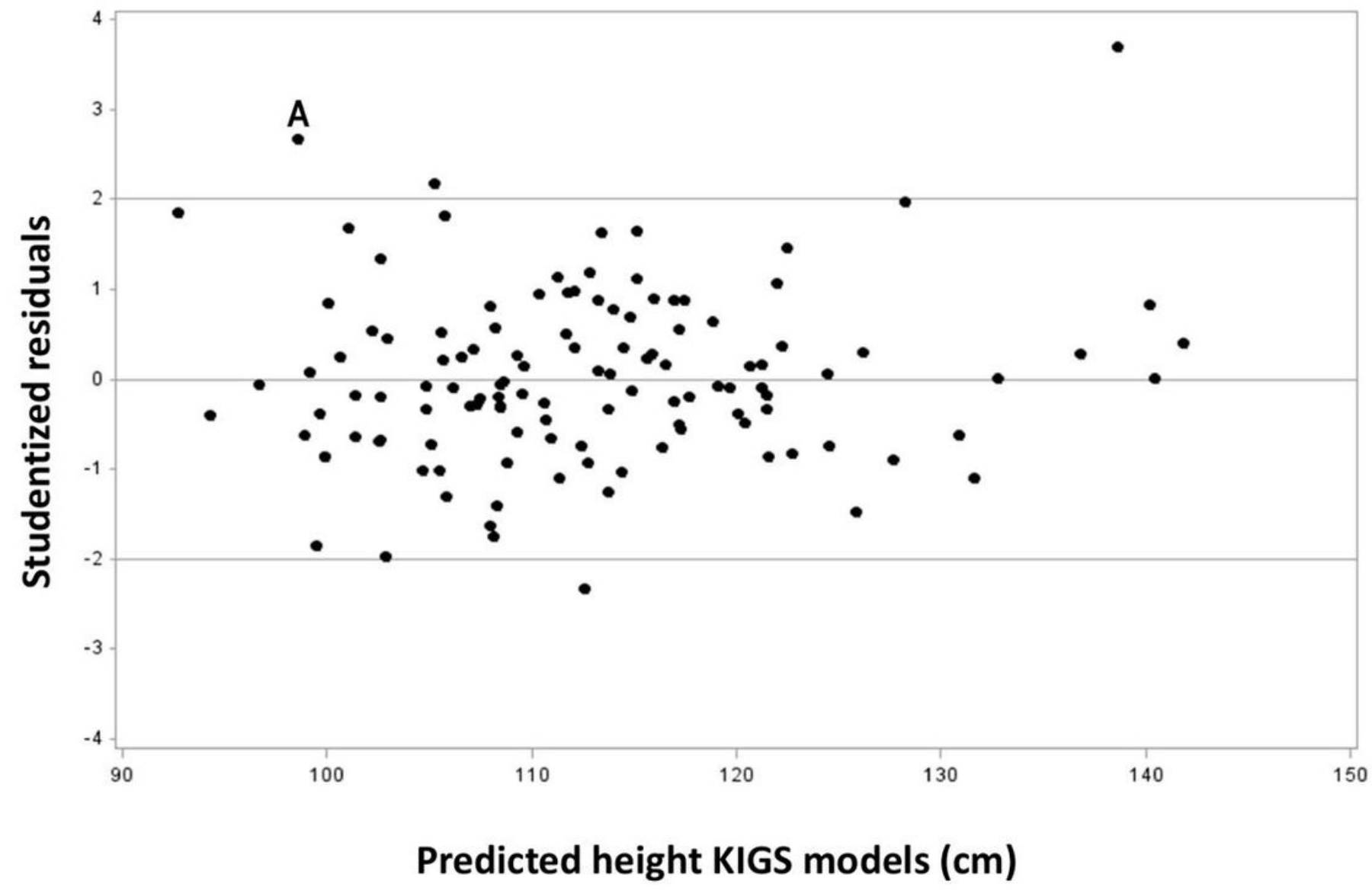

Figure 4

Individual studentized residuals in relation to first-year prediction according to the KIGS model. Patient A under-predicted with a high studentized residual. 


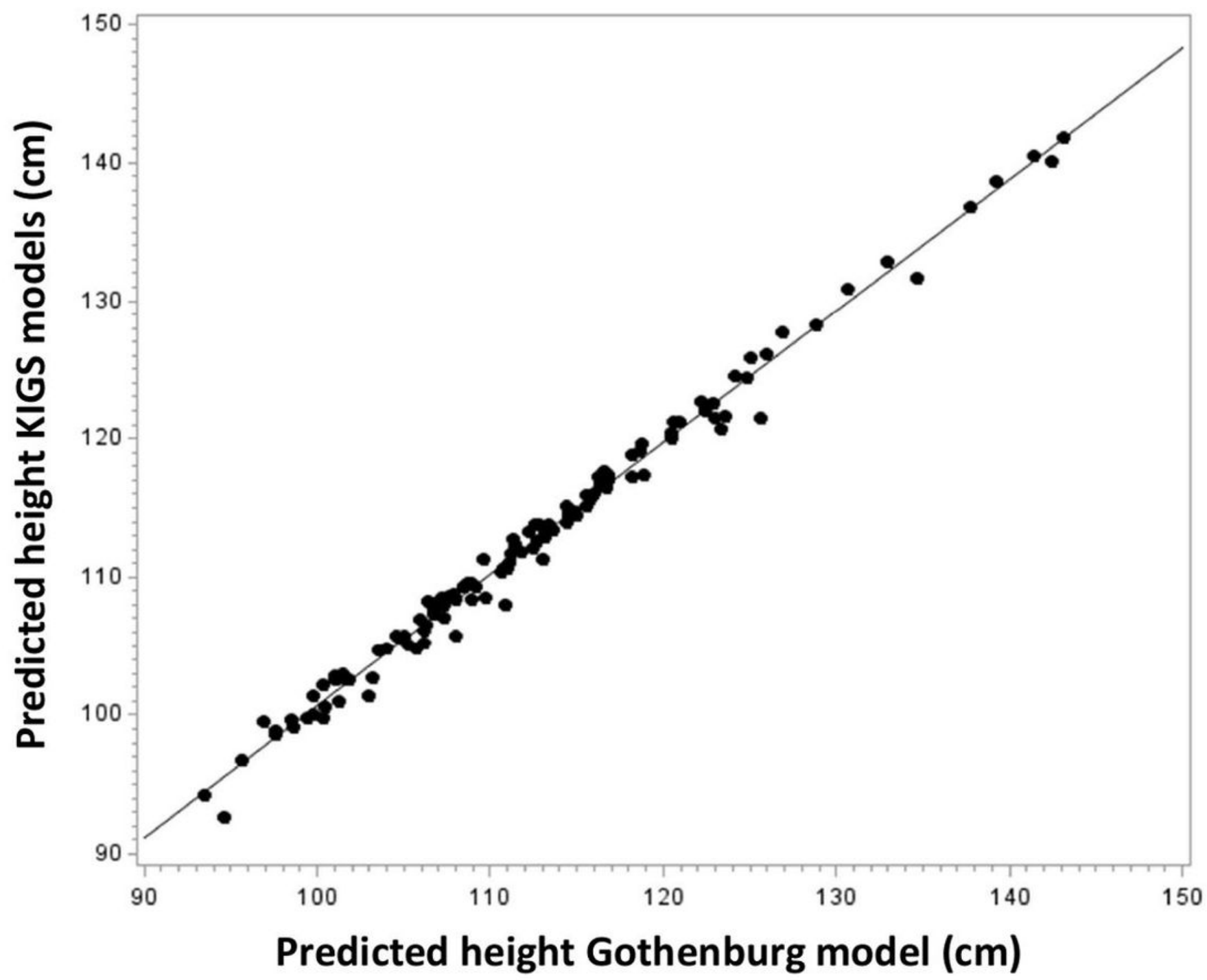

Figure 5

First-year height prediction according to the KIGS model compared to first-year height prediction according to the Gothenburg model shows a correlation of $r=0.995, p=<0.0001$. 